\title{
Quantum Character of Electromagnetic Langmuir Oscillations in Conventional Electron-Ion Plasma
}

\author{
Boris Alexandrovich Veklenko \\ Institute for High Energy of Joint Institute for High Temperature, Russian Academy of Science, Ijorskaja 13/19, Moscow 127412, Russia \\ Correspondence should be addressed to Boris Alexandrovich Veklenko, veklenkoba@yandex.ru
}

Received 22 January 2012; Revised 16 April 2012; Accepted 17 April 2012

Academic Editor: Adam Miranowicz

Copyright (c) 2012 Boris Alexandrovich Veklenko. This is an open access article distributed under the Creative Commons Attribution License, which permits unrestricted use, distribution, and reproduction in any medium, provided the original work is properly cited.

It is shown that the low-temperature plasma near-thermodynamic equilibrium cannot be classical because of a quantum nature of the longitudinal electromagnetic field and electron interaction with Rayleigh-Jeans distribution of Langmuir waves. The theory requires introduction of a dimensionless quantum charge whose value is greater than unity leading to a liquid-like behavior of the plasma.

\section{Introduction}

Plasma is called classical and the low-temperature one, if electrons and ions in it are governed by equations of classical electrodynamics and obey Maxwell distribution at thermodynamic equilibrium. The latter condition implies that the de-Brogle temperature wave length $\lambda_{B} \ll 1 / n^{1 / 3}$ is much less than the mean distance between the particles in plasma. Here, $n$ is the concentration of particles. The inequality written above does not mean that the electromagnetic field in plasma could be described also by classical physics methods. The latter is widely used as a traditional approach [1-5]. Describing the electromagnetic field with the help of the strength $\mathbf{E}(\mathbf{r}, t)$, we suppose that it is in a quantum coherent state [6] and the occupation numbers are much greater than unity. Although the latter is meaningful, the former has no grounds. Similarly, one could suppose that the field in plasma is in Fock quantum state with the mean value $\langle\widehat{\mathbf{E}}(\mathbf{r}, t)\rangle=0$. This is more preferable since there are the Fock states, which are generated by a thermal source. In any case, a classical electromagnetic field description of the plasma should be justified. The procedure of field quantization in plasma leads to the appearance of the quantum of Langmuir oscillations energy $\hbar \Omega$ with frequency $\Omega=\sqrt{e^{2} n / m}$. We use the rationalized Gauss unit system. Here, $e$ is electron charge, $m$ is its mass, and $\hbar$ is Planck constant. The quanta $\hbar \Omega$ do not play any role only if the classical energy of two-particle interaction at the distance of Debye radius $r_{D}=\sqrt{T / e^{2} n}$, where $T$ is the temperature, satisfying the inequality:

$$
\frac{e^{2}}{4 \pi r_{D}} \gg \hbar \Omega
$$

After substitution the explicit expressions for $r_{D}$ and $\Omega$, we get

$$
\frac{e^{2}}{4 \pi \hbar v_{e}} \gg 1,
$$

where $v_{e}=\sqrt{T / m}$ is the mean electron velocity in plasma. For $T \sim 10^{4} K^{0}$, it occurs that $v_{e} \sim 10^{7} \mathrm{~cm} / \mathrm{s}$ and $e^{2} / 4 \pi \hbar v_{e} \sim$ 10. At first glance, one could think that the inequality (2) is satisfied and the quantization procedure could be omitted. But, surprisingly, a dimensionless charge (2) appears in the theory, with the value greater than one. The analogous dimensionless charge $e^{2} / \hbar c=1 / 137$ is known in quantum electrodynamics which uses transversal electromagnetic waves as fine structure constant or Sommerfeld constant. Its small value permits there to use a perturbation technique. Basically, investigating the plasma, we deal with the same electrodynamics but dealing with the longitudinal waves. In this theory, the parameter denoting the vacuum light velocity $c$ is absent. It is replaced now by $v_{e} \ll c$. That is why 
the dimensionless charge (2) occurs being great and there appears a question about the applicability of the perturbation technique, which is the base of modern theory of plasma. We state that, in our case, the interaction constant is big and plasma has liquid-like but not a gaze structure. Moreover, the value of dimensionless charge (2) arises at $\hbar \rightarrow 0$ and the limiting transition to the conventional classical theory of plasma is not evident any more.

To resolve the problem, we develop a quantum field theory of plasma and investigate the conditions of the applicability of the classical theory.

\section{Principle Equations}

Let us consider the plasma in thermodynamic equilibrium in which the ions are uniformly distributed over the space forming a certain background, which, along with electrons, is neutral. We use the Heisenberg representation to describe the quantum electron field $\breve{\psi}(\mathbf{r}, t)$, where $\mathbf{r}$ is the spatial coordinate and $t$ is an arbitrary moment of time. Spin effects are omitted. Since the plasma electrons obey Maxwell distribution by definition, the results do not depend on the kind of statistics. Thus, we can use Bose-Einstein commutation relations:

$$
\left[\check{\psi}(x) ; \psi^{+}\left(x^{\prime}\right)\right]_{t=t^{\prime}}=\delta\left(\mathbf{r}-\mathbf{r}^{\prime}\right), \quad x=\{\mathbf{r}, t\} .
$$

The field operator $\check{\psi}(x)$ is described by the Schrodinger equation:

$$
i \hbar \frac{\partial \psi(\check{\psi}(x)}{\partial t}=\frac{1}{2 m}\left(\hat{p}^{2}-\frac{e}{c} \check{\mathbf{A}}(x)\right)^{2} \check{\psi}(x), \quad \hat{p}^{\nu}=-i \hbar \nabla^{v} .
$$

The electromagnetic field operator $\check{\mathrm{A}}(x)$ obeys the Maxwell equation:

$$
\nabla^{2} \check{A}^{v}(x)-\frac{1}{c^{2}} \frac{\partial^{2}}{\partial t^{2}} \check{A}^{\nu}(x)-\nabla^{\nu} \nabla^{\nu_{1}} \check{A}^{\nu_{1}}(x)=-\frac{1}{c} \check{j}^{\nu}(x),
$$

and the conventional commutation relation:

$$
\left[\check{A}^{\nu}(x) ; \frac{\partial}{\partial t^{\prime}} \check{A}^{\nu^{\prime}}\left(x^{\prime}\right)\right]_{t=t^{\prime}}=i \hbar c^{2} \delta_{\nu v^{\prime}} \delta\left(\mathbf{r}-\mathbf{r}^{\prime}\right) .
$$

We assume Einstein's summation rule over repeating indices $\nu$. The current density $\mathfrak{j}^{\nu}(x)$ is defined by expression:

$$
\begin{aligned}
\check{j}^{\nu}(x)= & \frac{e}{2 m} \check{\psi}^{+}(x) \hat{p}^{\nu} \check{\psi}(x) \\
& +H . c-\frac{e^{2}}{2 m c} \check{\psi}^{+}(x) \check{A}^{v}(x) \check{\psi}(x) .
\end{aligned}
$$

If the classical current $j_{\mathrm{cl}}^{\nu}(x)$ exists in the system, it is necessary to add it to $\check{j}^{v}(x)$. The system of $(4),(5),(7)$ is written in gauge condition with a zero scalar potential. It permits us to write the quantum Maxwell equations in a simple form (5)-(6). We are interested in the longitudinal electromagnetic field which is the potential field. That is why instead of zero scalar potential we introduce the pseudopotential $\check{\Phi}(x)$ by definition:

$$
\check{\mathbf{E}}(x)=-\frac{1}{c} \frac{\partial}{\partial t} \check{\mathbf{A}}(x)=-\nabla \check{\Phi}(x),
$$

or after Fourier transformation:

$$
\check{\Phi}(\mathbf{k}, \omega)=-\frac{\omega}{c k^{2}} k^{\nu} \check{A}^{\nu}(\mathbf{k}, \omega) .
$$

Further, it is convenient to exclude the vector potential Á $(x)$ from (4) in order to deal with the linear term $\check{\Phi}(x)$. Such a problem can be solved by using the auxiliary operator:

$$
\check{X}(x)=-\frac{1}{c} \int \check{A}^{v}(x) d l^{\nu} .
$$

The integration is performed over any curve connecting points $\mathbf{r}$ and $\mathbf{r}_{0}$, where $\mathbf{r}_{0}$ is any arbitrary point. By changing the variable:

$$
\check{\psi}(x)=e^{i(e / \hbar) \check{X}(x)} \stackrel{\check{\psi}}{\psi}(x)
$$

one can write (4) in the form:

$$
i \hbar \frac{\partial}{\partial t} \check{\widetilde{\psi}}(x)=\frac{\hat{p}^{2}}{2 m} \check{\widetilde{\psi}}(x)+e \check{\Phi}(x) \check{\widetilde{\psi}}(x)
$$

and write

$$
\check{j}^{\nu}(x)=\frac{e}{2 m} \widetilde{\psi}^{+}(x) \hat{p}^{\nu} \stackrel{\check{\psi}}{(x)+H . c .}
$$

instead of (7).

We omit tildes below. If $\check{\Phi}(x)$ is a classical function, we come to the conventional description of the classical plasma (see [7]). Below, we investigate how quantum longitudinal plasma waves affect the electrons. In a classical plasma, there are undamped Langmuir oscillations $[1,4]$ at the wave numbers $k<k_{c}=\Omega / v_{e}$. It means in connection with commutations relations (6) that

$$
\begin{gathered}
\check{A}^{\nu}(x)=\sum_{\mathbf{k} \lambda} \sqrt{\frac{\hbar c^{2}}{2 V \omega(\lambda)}} e_{\mathbf{k} \lambda}^{v}\left(\hat{\alpha}_{\mathbf{k} \lambda} e^{i \mathbf{k r}-i \omega(\lambda) t}+\hat{\alpha}_{\mathbf{k} \lambda}^{+} e^{-i \mathbf{k r}+i \omega(\lambda) t}\right), \\
\omega(\lambda)= \begin{cases}\omega(k) & \text { if } \lambda=1,2, \\
\Omega \vartheta\left(k_{c}-k\right) & \text { if } \lambda=3 .\end{cases}
\end{gathered}
$$

Here, $\hat{\alpha}_{\mathbf{k} \lambda}$ and $\hat{\alpha}_{\mathbf{k} \lambda}^{+}$are the annihilation and the creation Langmuir's wave operators with vector $\mathbf{k}$ and polarization index $\lambda$. For the transversal waves $\lambda=1,2$, for the longitudinal one, $\lambda=3, \vartheta\left(k_{c}-k\right)$ is the Heaviside step function. The longitudinal part of vector potential satisfies the equation:

$$
-\frac{\partial^{2}}{c^{2} \partial t^{2}} \check{A}^{v}(x)-\frac{\Omega^{2}}{c^{2}} \check{A}^{v}(x)=0 \quad \text { at } k<k_{c} .
$$

Now, from (9), one gets

$$
\begin{aligned}
\check{\Phi}(x) & =-\sum_{\mathbf{k}} \sqrt{\frac{\hbar \Omega}{2 V k^{2}}}\left(\hat{\alpha}_{\mathbf{k} 3} e^{i \mathbf{k r}-i \Omega t}+\hat{\alpha}_{\mathbf{k} 3}^{+} e^{-i \mathbf{k r}+i \Omega t}\right) \vartheta\left(k_{c}-k\right) \\
& =\Phi^{(+)}(x)+\Phi^{\check{(}-)}(x) .
\end{aligned}
$$


The function $\varrho(k)$ deals with transversal waves fall out of calculations. We shall use below some auxiliary constructions. strait calculation yields

$$
\begin{aligned}
\left\langle\Phi^{\check{(}-)}(x) \Phi^{\check{(+)}}\left(x^{\prime}\right)\right\rangle= & \sum_{\mathbf{k}} \frac{\hbar \Omega}{2 k^{2}} e^{i \mathbf{k}\left(\mathbf{r}-\mathbf{r}^{\prime}\right)-i \Omega\left(t-t^{\prime}\right)} \frac{\vartheta\left(k_{c}-k\right)}{e^{\hbar \Omega / T}-1}, \\
\left\langle\Phi^{\check{(}+)}(x) \Phi^{\check{(}-)}\left(x^{\prime}\right)\right\rangle= & \sum_{\mathbf{k}} \frac{\hbar \Omega}{2 k^{2}} e^{i \mathbf{k}\left(\mathbf{r}-\mathbf{r}^{\prime}\right)-i \Omega\left(t-t^{\prime}\right)} \\
& \times\left[1+\frac{1}{e^{\hbar \Omega / T}-1}\right] \vartheta\left(k_{c}-k\right) .
\end{aligned}
$$

The averaging in formulae (17) performs both in quantum and statistical senses over Gibbs distribution, and if $\hbar \Omega \ll T$, then (Rayleigh-Jeans approximation)

$$
\left\langle\Phi^{\check{(-)}(x)} \Phi^{\check{(+)}}(x)\right\rangle=\left\langle\Phi^{\check{(+)}}(x) \Phi^{\check{(-)}(x)}\right\rangle=\frac{T}{4 \pi^{2}} \frac{\Omega}{v_{e}} .
$$

\section{Green Functions $G_{r}\left(x, x^{\prime}\right)$ and $G_{a}\left(x, x^{\prime}\right)$}

For investigation of the equation system (5)-(16) it is convenient to use the method of Green functions. Let by definition

$$
\begin{aligned}
G_{r}\left(x, x^{\prime}\right) & =\frac{1}{i \hbar}\left\langle\left[\check{\psi}(x) ; \check{\psi}^{+}\left(x^{\prime}\right)\right]\right\rangle \vartheta\left(t-t^{\prime}\right), \\
G_{a}\left(x, x^{\prime}\right) & =-\frac{1}{i \hbar}\left\langle\left[\check{\psi}(x) ; \check{\psi}^{+}\left(x^{\prime}\right)\right]\right\rangle \vartheta\left(t^{\prime}-t\right) .
\end{aligned}
$$

Using (12), we get

$$
\begin{aligned}
i \hbar \frac{\partial}{\partial t} G_{r}\left(x, x^{\prime}\right)= & \delta\left(x, x^{\prime}\right)+\frac{\hat{p}^{2}}{2 m} G_{r}\left(x, x^{\prime}\right) \\
& -\frac{i e}{\hbar}\left\langle\left[\check{\Phi}(x) \check{\psi}(x) ; \check{\psi}^{+}\left(x^{\prime}\right)\right]\right\rangle \vartheta\left(t-t^{\prime}\right) .
\end{aligned}
$$

Using (12) again, we have for the right term in (20)

$$
\begin{aligned}
\frac{\partial}{\partial t}\langle[ & \left.\left.\check{\Phi}(x) \check{\psi}(x) ; \check{\psi}^{+}\left(x^{\prime}\right)\right]\right\rangle \vartheta\left(t-t^{\prime}\right) \\
= & \left\langle\left[\frac{\partial \check{\Phi}(x)}{\partial t} \check{\psi}(x) ; \check{\psi}^{+}\left(x^{\prime}\right)\right]\right\rangle \vartheta\left(t-t^{\prime}\right) \\
& +\frac{1}{i \hbar}\left\langle\left[\check{\Phi}(x) \frac{\hat{p}^{2}}{2 m} \check{\psi}(x) ; \check{\psi}^{+}\left(x^{\prime}\right)\right]\right\rangle \vartheta\left(t-t^{\prime}\right) \\
& +\frac{e}{i \hbar}\left\langle\left[\check{\Phi}(x) \check{\Phi}(x) \check{\psi}(x) ; \check{\psi}^{+}\left(x^{\prime}\right)\right]\right\rangle \vartheta\left(t-t^{\prime}\right) .
\end{aligned}
$$

In order to get the closed equation system, we use the following conventional procedure of breaking up the correlators in (21):

$$
\begin{aligned}
\left\langle\left[\check{\Phi}(x) \check{\Phi}(x) \check{\psi}(x) ; \check{\psi}^{+}\left(x^{\prime}\right)\right]\right\rangle \vartheta\left(t-t^{\prime}\right) \\
=\langle\check{\Phi}(x) \check{\Phi}(x)\rangle\left\langle\left[\check{\psi}(x) ; \check{\psi}^{+}\left(x^{\prime}\right)\right]\right\rangle \\
\quad \times \vartheta\left(t-t^{\prime}\right) .
\end{aligned}
$$

Such breaking is certainly correct if the fields do not interact or the interaction is sufficiently weak. In practice, the region of its validity is much greater. Its accuracy is greater than the accuracy of a conventional Hartree-Fock approximation when one supposes the correlator braking in the first (20) equation of system. But now, one breaks the correlators in the second equation (21) of the system. In some cases by such a way, one can develop a theory of phase transitions [8]. If $\hbar \Omega \ll T$, one can suppose that $\check{\Phi} \hat{p}^{2} \breve{\psi} \approx \hat{p}^{2} \check{\Phi} \check{\psi}$. Now, (21)-(22) could be easily solved using Fourier transformation:

$$
\begin{aligned}
\left\langle\left[\Phi^{\left.\check{(+)} \check{\psi} ; \check{\psi}^{+}\right]}\right\rangle_{\mathbf{p} E}=\right. & \frac{e\left\langle\Phi^{\check{(+)}}(x) \Phi^{\check{(-)}}(x)\right\rangle}{(-i / \hbar) E+i \Omega+\left(i p^{2} / 2 m \hbar\right)} \\
& \times G_{r}\left(\frac{\mathbf{p}}{\hbar}, \frac{E}{\hbar}\right), \\
\left\langle\left[\Phi^{(\check{(})} \check{\psi} ; \check{\psi}^{+}\right]\right\rangle_{\mathbf{p} E}= & \frac{e\left\langle\Phi^{\check{(}-)}(x) \Phi^{\check{(+)}}(x)\right\rangle}{(-i / \hbar) E-i \Omega+\left(i p^{2} / 2 m \hbar\right)} \\
& \times G_{r}\left(\frac{\mathbf{p}}{\hbar}, \frac{E}{\hbar}\right) .
\end{aligned}
$$

Finally, from (20), one gets

$$
\begin{aligned}
G_{r}\left(\frac{\mathbf{p}}{\hbar}, \frac{E}{\hbar}\right)= & {\left[E+i 0-\frac{p^{2}}{2 m}-2 e^{2}\left\langle\Phi^{\check{(+)}}(x) \Phi^{\check{(-)}}(x)\right\rangle\right.} \\
& \left.\times \frac{E-\left(p^{2} / 2 m\right)}{\left(E-\left(p^{2} / 2 m\right)+i 0\right)^{2}-\hbar^{2} \Omega^{2}}\right]^{-1} .
\end{aligned}
$$

In accordance with (16), we took into account that

$$
\begin{gathered}
\frac{\partial}{\partial t} \Phi^{\check{(+)}(x)}=-i \Omega \Phi^{\check{(+)}}(x), \\
\frac{\partial}{\partial t} \Phi^{\check{(-)}}(x)=i \Omega \Phi^{\check{(-)}(x) .}
\end{gathered}
$$

The function $G_{r}(\mathbf{p} / \hbar, E / \hbar)$ is analytical in upper half plane of complex $E$ by definition due to Heaviside step function $\vartheta\left(t-t^{\prime}\right)$. That is why the term $i 0$ appears in (24).

In accordance with (24), the interaction of plasma electrons with thermal quantum electromagnetic fields is described by a pole leading to qualitative deformation of their properties. Formula (24), in accordance with (18), can be rewritten as

$$
G_{r}\left(\frac{\mathbf{p}}{\hbar}, \frac{E}{\hbar}\right)=\frac{\left(E-\left(p^{2} / 2 m\right)\right)^{2}-\hbar^{2} \Omega^{2}}{\left(E-E^{(0)}(\mathbf{p})\right)\left(E-E^{(1)}(\mathbf{p})\right)\left(E-E^{(2)}(\mathbf{p})\right)}
$$


where

$$
\begin{aligned}
E^{(0)}(\mathbf{p}) & =\frac{p^{2}}{2 m} E^{(1)}, \\
E^{(1)}(\mathbf{p}) & =\frac{p^{2}}{2 m}+\hbar \Omega \sqrt{1+Z}, \\
E^{(2)}(\mathbf{p}) & =\frac{p^{2}}{2 m}-\hbar \Omega \sqrt{1+Z}, \\
Z & =\frac{1}{2 \pi^{2}}\left(e^{*}\right)^{2} \frac{T}{\hbar \Omega}=\frac{1}{(2 \pi)^{3}} \frac{r_{D}}{r_{B}}, \\
\left(e^{*}\right)^{2} & =\frac{e^{2}}{\hbar v_{e}},
\end{aligned}
$$

and $r_{B}=4 \pi \hbar^{2} / e^{2} m$ is the Bohr radius of Hydrogen atom. So, the procedure of quantization of the longitudinal electromagnetic field leads to the emergence of the dimensionless charge $e^{*}$ introduced in the introduction qualitatively. Formula (26) can be rewritten in a form:

$$
\begin{aligned}
G_{r}\left(\frac{\mathbf{p}}{\hbar}, \frac{E}{\hbar}\right)= & \frac{1}{1+Z} \frac{1}{E-\left(p^{2} / 2 m\right)+i 0} \\
& +\frac{Z}{2(1+Z)} \frac{1}{E-\left(p^{2} / 2 m\right)-\hbar \Omega \sqrt{1+Z}+i 0} \\
& +\frac{Z}{2(1+Z)} \frac{1}{E-\left(p^{2} / 2 m\right)+\hbar \Omega \sqrt{1+Z}+i 0} .
\end{aligned}
$$

The limit $Z \rightarrow 0$ corresponds to free electrons without field $\check{\Phi}$. If $Z \gg 1$, the free electrons are replaced by two types of quasi-particles described by the two last terms of (28). It is the first result of present paper. Namely, these quasi particles describe electrodynamics, thermodynamic, and especially kinetic properties of plasma at $Z \gg 1$. Since such inequality is always satisfied for low-temperature plasma, the quantum parameter $Z>1$ should be present in all formulae. Nevertheless, one should stress that the used formalism and, in particular, the procedure of correlator breaking does not guarantee the accuracy of quantitative results at $Z \gg 1$. For this reason, the extrapolation of the results to the region $Z \gg 1$ is expected to lead as in theory of liquids to semiquantitative predictions. But at the same time, this way proves that theories which use limit $Z \rightarrow 0$ (Vlasov equation) are not correct.

It follows from formula (28) that at $Z \gg 1$ there exists any "camouflage effect" that sometimes hides the Plank's constant $\hbar$. For instance, in such region

$$
\begin{aligned}
G_{r}\left(\frac{\mathbf{p}}{\hbar}, \frac{E}{\hbar}\right)= & \frac{1}{2} \frac{1}{E-\left(p^{2} / 2 m\right)-\hbar \Omega \sqrt{Z}+i 0} \\
& +\frac{1}{2} \frac{1}{E-\left(p^{2} / 2 m\right)+\hbar \Omega \sqrt{Z}+i 0},
\end{aligned}
$$

and Plank's constant disappeared since using explicit form (27) for $Z$, we get

$$
\hbar \Omega \sqrt{Z}=\sqrt{\frac{e^{2}}{2 \pi^{2} r_{D}} T}, \quad Z \gg 1 .
$$

That is why, in this region, the erroneous picture of electron clouds as an ensemble of free electrons sometimes leads to the pseudo reasonable results following, in particular, from Vlasov equation [2].

\section{Chemical Potential of Electron Gas}

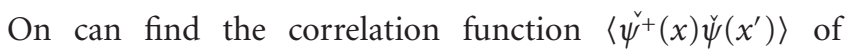
electron gas with the help of Green functions $G_{r, a}$ and fluctuation-dissipation theorem [9]:

$$
\begin{aligned}
\left\langle\check{\psi}^{+}(x) \check{\psi}\left(x^{\prime}\right)\right\rangle=\frac{i}{V} \sum_{\mathbf{p}} & \int \frac{1}{e^{(E-\mu / T)}-1} \\
& \times\left[G_{r}\left(\frac{\mathbf{p}}{\hbar}, \frac{E}{\hbar}\right)-G_{a}\left(\frac{\mathbf{p}}{\hbar}, \frac{E}{\hbar}\right)\right] \\
& \times e^{(i / \hbar) \mathbf{p}\left(\mathbf{r}-\mathbf{r}^{\prime}\right)-(i / \hbar) E\left(t-t^{\prime}\right)} \frac{d E}{2 \pi} .
\end{aligned}
$$

For nondegenerate gas,

$$
\begin{gathered}
e^{(E-\mu) / T} \gg 1, \\
\left\langle\check{\psi}^{+}(x) \check{\psi}(x)\right\rangle \\
=\frac{i}{V} \sum_{\mathbf{p}} \int e^{-(E-\mu) / T}\left[G_{r}\left(\frac{\mathbf{p}}{\hbar}, \frac{E}{\hbar}\right)-G_{a}\left(\frac{\mathbf{p}}{\hbar}, \frac{E}{\hbar}\right)\right] \frac{d E}{2 \pi} .
\end{gathered}
$$

One gets from (28) that

$$
\begin{aligned}
G_{r}\left(\frac{\mathbf{p}}{\hbar}, \frac{E}{\hbar}\right)-G_{a}\left(\frac{\mathbf{p}}{\hbar}, \frac{E}{\hbar}\right)= & -\frac{2 \pi i}{1+Z} \delta\left(E-E^{(0)}\right) \\
& -i \pi \frac{Z}{1+Z} \delta\left(E-E^{(1)}\right) \\
& -i \pi \frac{Z}{1+Z} \delta\left(E-E^{(2)}\right) .
\end{aligned}
$$

Since

$$
n=\left\langle\check{\psi}^{+}(x) \check{\psi}(x)\right\rangle,
$$

one has the equation for chemical potential which is easily solved

$$
e^{-\left(\mu-\mu_{0}\right) / T}=\frac{1}{1+Z}+\frac{Z}{1+Z} \operatorname{ch} \frac{\hbar \Omega \sqrt{1+Z}}{T},
$$

where

$$
\mu_{0}=-T \ln \frac{1}{n}\left(\frac{m T}{2 \pi \hbar^{2}}\right)^{3 / 2}
$$

is the chemical potential of ideal electron gas. Formula (36) possesses a remarkable property. The difference $\mu-\mu_{0}$ in both regions as $Z \ll 1$ and $Z \gg 1$ according to equality $\hbar \Omega \sqrt{Z}=$ $\sqrt{T e^{2} / 2 \pi^{2} r_{D}}$, that is,

$$
\begin{aligned}
e^{-\left(\mu-\mu_{0}\right) / T} & =\operatorname{ch} \frac{\hbar \Omega \sqrt{Z}}{T}=c h \sqrt{\frac{e^{2}}{2 \pi^{2} r_{D} T}} \\
& =1-\frac{e^{2}}{4 \pi^{2} r_{D} T} \quad \text { if } \frac{e^{2}}{r_{D} T} \ll 1, \quad Z \gg 1,
\end{aligned}
$$


does not depend on Planck constant. So only for $Z \sim 1$ this difference depends on $\hbar$. We remark that in plasma always $e^{2} / r_{D} T \ll 1$. For typical values of parameters, $n \sim$ $10^{19} 1 / \mathrm{cm}^{3}$ and $T \sim 10^{4} K^{0}$, it turns out that $Z \sim 50, T / \hbar \Omega \sim$ $10, \hbar \Omega \sqrt{1+Z} / T \sim 1$. If the concentration $n$ diminishes then $Z$ increases but the ratio $\hbar \Omega \sqrt{1+Z} / T$ diminishes. So at higher concentration $n$ the procedure of electromagnetic field quantization according to (36) leads to the violation of the Sacha formula:

$$
\frac{x}{1-x^{2}}=n\left(\frac{m T}{2 \pi \hbar^{2}}\right)^{3 / 2} e^{-I / T}\left(\frac{1}{1+Z}+\frac{Z}{1+Z} \operatorname{ch} \frac{\hbar \Omega \sqrt{1+Z}}{T}\right),
$$

where $I$ is the ionization potential. The corrector term unclosed in brackets depends on $\hbar$ only if $Z$ is of the order of unity.

\section{Plasma Dielectric Permittivity for Longitudinal Waves}

Let the classical current $j_{\mathrm{cl}}^{v}(x)$ generating the classical potential $\varphi_{\mathrm{cl}}(x)$ exists in plasma. Now, (12) takes the view:

$$
i \hbar \frac{\partial}{\partial t} \check{\widetilde{\psi}}(x)=\frac{\hat{p}^{2}}{2 m} \check{\widetilde{\psi}}(x)+e\left(\check{\Phi}(x)+\varphi_{\mathrm{cl}}(x)\right) \check{\widetilde{\psi}}(x) .
$$

This equation can be rewritten in integral form:

$$
\check{\psi}(x)=\check{\psi}^{0}(x)+\frac{e}{i \hbar} \int\left[\check{\psi}(x) ; \check{\psi}^{+}\left(x^{\prime}\right)\right] \varphi_{\mathrm{cl}}\left(x^{\prime}\right) \check{\psi}\left(x^{\prime}\right) d x^{\prime},
$$

where $\check{\psi}^{0}(x)$ is the solution of $(40)$ at $\varphi_{\mathrm{cl}}(x)=0$. We use approximation:

$$
\begin{aligned}
\frac{e}{i \hbar}\left[\check{\psi}(x) ; \check{\psi}^{+}\left(x^{\prime}\right)\right] \vartheta\left(t-t^{\prime}\right) \approx & \frac{e}{i \hbar}\left\langle\left[\check{\psi}(x) ; \check{\psi}^{+}\left(x^{\prime}\right)\right]\right\rangle \\
& \times \vartheta\left(t-t^{\prime}\right)=G_{r}\left(x ; x^{\prime}\right) .
\end{aligned}
$$

The electron density at first approximation over the potential $\varphi_{\mathrm{cl}}(x)$ is

$$
n(x)=\left\langle\check{\psi}^{+}(x) \check{\psi}(x)\right\rangle=\left\langle\psi^{\check{0}+}(x) \check{\psi}^{0}(x)\right\rangle+\delta n(x),
$$

where

$$
\delta n(x)=e \int G_{r}^{*}\left(x, x_{1}\right) \varphi_{\mathrm{cl}}\left(x_{1}\right)\left\langle\check{\psi}^{0+}\left(x_{1}\right) \check{\psi}^{0}(x)\right\rangle d x_{1}+\text { c.c. }
$$

According to (33) and (34), one gets

$$
\begin{aligned}
\left\langle\psi^{\breve{0}+}\left(x_{1}\right) \breve{\psi}^{0}(x)\right\rangle=\frac{1}{V} \sum_{\mathbf{p}} \int & e^{(i / \hbar) \mathbf{p}\left(\mathbf{r}-\mathbf{r}_{1}\right)-(i / \hbar) E\left(t-t_{1}\right)} e^{-(E-\mu) / T} \\
\times & {\left[\frac{1}{1+Z} \delta\left(E-E^{(0)}\right)\right.} \\
& +\frac{Z}{2(1+Z)} \delta\left(E-E^{(1)}\right) \\
& \left.+\frac{Z}{2(1+Z)} \delta\left(E-E^{(2)}\right)\right] d E .
\end{aligned}
$$

Now, the increment of the electron concentration in plasma $\delta n(x)$ as a function of $x$ due to their interaction with field $\varphi_{\mathrm{cl}}(x)$ can be written as

$$
\begin{aligned}
\delta n(x)=\frac{e}{V^{2}} \sum_{\mathbf{p} \mathbf{p}_{1} \mathbf{k}} \int \delta\left(\frac{\mathbf{p}_{1}}{\hbar}-\frac{\mathbf{p}}{\hbar}+\mathbf{k}\right) e^{i \mathbf{k r}-i \omega t+(\mu / T)} \\
\times\left[\frac{1}{1+Z} G_{r}\left(\frac{\mathbf{p}}{\hbar}, \frac{E^{(0)}\left(\mathbf{p}_{1}\right)+\hbar \omega}{\hbar}\right) \exp \left(-\frac{E^{(0)}\left(\mathbf{p}_{1}\right)}{T}\right)\right. \\
+\frac{Z}{2(1+Z)} G_{r}\left(\frac{\mathbf{p}}{\hbar}, \frac{E^{(1)}\left(\mathbf{p}_{1}\right)+\hbar \omega}{\hbar}\right) \\
\\
\quad \times \exp \left(-\frac{E^{(1)}\left(\mathbf{p}_{1}\right)}{T}\right)+\frac{Z}{2(1+Z)} \\
\left.\quad \times G_{r}\left(\frac{\mathbf{p}}{\hbar}, \frac{E^{(2)}\left(\mathbf{p}_{1}\right)+\hbar \omega}{\hbar}\right) \exp \left(-\frac{E^{(2)}\left(\mathbf{p}_{1}\right)}{T}\right)\right] \\
\times \varphi_{\mathrm{cl}}(\mathbf{k}, \omega) \frac{d \omega}{2 \pi}+c . c,
\end{aligned}
$$

where

$$
\varphi_{\mathrm{cl}}(\mathbf{k}, \omega)=\int e^{i \mathbf{k r}-i \omega t} \varphi_{\mathrm{cl}}(\mathbf{r}, t) d \mathbf{r} d t
$$

The plasma dielectric permittivity can be found from (5) and equation of discontinuity:

$$
\omega e \delta n(\mathbf{k}, \omega)-k^{\nu} j_{\mathrm{cl}}^{\nu}(\mathbf{k}, \omega)=0 .
$$

On the other hand, from the mean value equality $-\partial \mathrm{A} / c \partial t=-\nabla \varphi_{\mathrm{cl}}$, it follows that $\omega k^{\nu} A^{\nu}=-c k^{2} \varphi_{\mathrm{cl}}$. After Fourier transformation of (5), multiplying the result by $k^{\nu}$ and taking into account the presence of classical current and longitudinal waves, one gets

$$
\frac{\omega^{2}}{c^{2}} k^{\nu} A^{\nu}(\mathbf{k}, \omega)=-\frac{\omega}{c} e \delta n(\mathbf{k}, \omega)-\frac{1}{c} k^{\nu} j_{\mathrm{cl}}^{\nu}(\mathbf{k}, \omega) .
$$

After comparing this expression with the definition of dielectric permittivity $\varepsilon^{l}(\mathbf{k}, \omega)$ :

$$
\frac{\omega^{2}}{c^{2}} \varepsilon^{l}(\mathbf{k}, \omega) k^{v} A^{v}(\mathbf{k}, \omega)=-\frac{1}{c} k^{v} j_{\mathrm{cl}}^{\nu}(\mathbf{k}, \omega),
$$

one gets

$$
\varepsilon^{l}(\mathbf{k}, \omega)=1+e \frac{c}{\omega} \frac{\delta n(\mathbf{k}, \omega)}{k^{v} A^{v}(\mathbf{k}, \omega)} .
$$

Now,

$$
\begin{aligned}
\mathcal{E}^{l}(\mathbf{k}, \omega)= & 1-\frac{e^{2}}{k^{2} V} \sum_{\mathbf{p}}\left[\frac{1}{1+Z} G_{r}\left(\frac{\mathbf{p}+\hbar \mathbf{k}}{\hbar}, \frac{E^{(0)}+\hbar \omega}{\hbar}\right) e^{-E^{(0)} / T}\right. \\
& +\frac{Z}{2(1+Z)} G_{r}\left(\frac{\mathbf{p}+\hbar \mathbf{k}}{\hbar}, \frac{E^{(1)}+\hbar \omega}{\hbar}\right) e^{-E^{(1)} / T} \\
& \left.+\frac{Z}{2(1+Z)} G_{r}\left(\frac{\mathbf{p}+\hbar \mathbf{k}}{\hbar}, \frac{E^{(2)}+\hbar \omega}{\hbar}\right) e^{-E^{(2)} / T}\right] e^{\mu / T} \\
& + \text { H.c. }
\end{aligned}
$$


H.c. means the complex conjugate values and simultaneously changing $\mathbf{k} \rightarrow-\mathbf{k}$ and $\omega \rightarrow-\omega$.

The explicit form of the dielectric permittivity one can obtain from (52) and (28) if simultaneously one takes into account that for not too large $k$ with respect to $k<k_{c}=$ $\Omega / v_{e}$ the inequalities $\hbar^{2} k^{2} / 2 m \ll \hbar \Omega \sqrt{1+Z}$ and $\hbar k \ll p$ take place:

$$
\begin{aligned}
\mathcal{E}^{l}(\mathbf{k}, \omega)=1- & \frac{e^{2}}{k^{2} V} \sum_{\mathbf{p}} e^{-\left(E^{(0)}-\mu\right) / T} \\
\times & \left\{\frac{k^{2}}{m(\omega-(\mathbf{p k} / m)+i 0)^{2}}\right. \\
& \times\left[\frac{1}{(1+Z)^{2}}+\frac{1}{2}\left(\frac{Z}{1+Z}\right)^{2} \operatorname{ch} \frac{\hbar \Omega \sqrt{1+Z}}{T}\right] \\
& +\frac{2 Z}{\hbar(1+Z)^{2}} \\
& \times \frac{\Omega \sqrt{1+Z} \operatorname{sh}(\hbar \Omega \sqrt{1+Z / T})+\left(\hbar k^{2} / m\right)}{(\omega-(\mathbf{p k} / m)+i 0)^{2}-\Omega^{2}(1+Z)} \\
& +\frac{2}{\hbar}\left(\frac{Z}{1+Z}\right)^{2} \\
& \left.\times \frac{\Omega \sqrt{1+Z} \operatorname{sh}(\hbar \Omega \sqrt{1+Z / T})}{(\omega-(\mathbf{p k} / m)+i 0)^{2}-4 \Omega^{2}(1+Z)}\right\}
\end{aligned}
$$

From this formula, it follows that there are three resonance regions in plasma: $\omega \sim 0$-the Vlasov-Landau region at small frequencies and the new resonance regions in vicinities of quantum frequencies $\omega \sim \Omega \sqrt{1+Z}$ and $\omega \sim 2 \Omega \sqrt{1+Z}$. The existence of these regions manifests themselves in a characteristic way in the imaginary part of the dielectric permittivity. From (53), one gets $(\omega>0)$ :

$$
\begin{aligned}
\operatorname{Im} \varepsilon^{l}(\mathbf{k}, \omega)=\pi & \frac{e^{2} m n \omega}{k^{3} T \sqrt{2 \pi m T}} e^{\left(\mu-\mu_{0}\right) / T} \\
& \times\left\{\left[\frac{1}{(1+Z)^{2}}+\frac{1}{2}\left(\frac{Z}{1+Z}\right)^{2} c h \frac{\hbar \Omega \sqrt{1+Z}}{T}\right]\right. \\
& \times e^{-(\omega / k)^{2}(m / 2 T)}+\frac{Z}{(1+Z)^{2}} \frac{T}{\hbar \omega} \\
& \times e^{-((\omega-\Omega \sqrt{1+Z}) / k)^{2}(m / 2 T)} \\
& \times \frac{\Omega \sqrt{1+Z} s h(\hbar \Omega \sqrt{1+Z / T})+\left(\hbar k^{2} / m\right)}{\Omega \sqrt{1+Z}} \\
& +\left(\frac{Z}{1+Z}\right)^{2} \frac{T}{2 \hbar \omega} e^{-((\omega-2 \Omega \sqrt{1+Z}) / k)^{2}(m / 2 T)} \\
& \left.\times s h \frac{\hbar \Omega \sqrt{1+Z}}{T}\right\} .
\end{aligned}
$$

From this equality at $Z \rightarrow 0$, as expected, one finds the Landau attenuation. As the parameter $Z$ goes up, the plasma starts to absorb at frequencies $\omega \sim \Omega \sqrt{1+Z}$. Then, this effect disappears. And if $Z \gg 1$,

$$
\begin{aligned}
\operatorname{Im} \varepsilon^{l}(\mathbf{k}, \omega)=\pi & \frac{e^{2} m n \omega}{k^{3} T \sqrt{2 \pi m T}} e^{\left(\mu-\mu_{0}\right) / T} \\
\times[ & \frac{1}{2} e^{-(\omega / k)^{2}(m / 2 T)} \operatorname{ch} \frac{\hbar \Omega \sqrt{Z}}{T} \\
& +\frac{T}{2 \hbar \omega} e^{-((\omega-2 \Omega \sqrt{Z}) / k)^{2}(m / 2 T)} \\
& \left.\times \operatorname{sh} \frac{\hbar \Omega \sqrt{Z}}{T}\right] .
\end{aligned}
$$

In other words, the plasma absorbs at $Z \gg 1$ not only at low frequencies, but at high quantum frequencies $\omega \sim 2 \Omega \sqrt{Z}=2 \sqrt{T e^{2} / 2 \pi^{2} r_{D}} / \hbar$ as well. At $Z \gg 1$ and low frequencies, $\operatorname{Im} \varepsilon^{l}(\mathbf{k}, \omega)$ is a half of the imagine part of permittivity predicted by Landau theory [3] at $Z=0$. The intensity of absorption at the frequencies $\omega \sim 2 \Omega \sqrt{Z} \sim$ $1 / \hbar$ is, respectively, larger than intensity of low frequencies absorption.

Since

$$
\operatorname{Im} \varepsilon^{l}(\mathbf{k}, \omega)=\frac{1}{\omega} \sigma^{l}(\mathbf{k}, \omega),
$$

where $\sigma^{l}(\mathbf{k}, \omega)$ is the conductivity of substance one states that the parameter $Z$ plays an important role in kinetic properties of plasma.

As for the real part of dielectric permittivity at $Z=0$, (52) shows the conventional results, and in particular the Vlasov dispersion formula [2]:

$$
\omega^{2}=\Omega^{2}\left(1+3 k^{2} r_{D}^{2}\right), \quad k^{2} r_{D}^{2} \ll 1 .
$$

We remind that really $Z>>1$. If $Z$ grows, but still it is small, the conventional results of Vlasov theory change considerably. Let for the beginning $k^{2} r_{D}^{2} \ll Z \ll 1$. The explicit form of equation $\operatorname{Re} \varepsilon^{L}(\mathbf{k}, \omega)=0$ describing oscillation spectrum of the system in linear approximation over $Z$ and by $k v_{e} \omega \ll\left|\omega^{2}-\Omega^{2}(1+Z)\right|$ has the form:

$$
1-\frac{B_{1}(k, \omega, Z)}{\omega^{2}}-\frac{B_{2}(k, \omega, Z)}{\omega^{2}-\Omega^{2}(1+Z)}=0,
$$

where

$$
\begin{aligned}
B_{1}(k, \omega, Z)= & \Omega^{2}\left(1+3 \frac{T k^{2}}{\omega^{2} m}\right) \\
& \times(1-Z) e^{\left(\mu-\mu_{0}\right) / T}, \quad 1 \gg 3 \frac{T k^{2}}{\omega^{2} m}, \\
B_{2}(k, \omega, Z)= & \Omega^{2} \frac{2 Z}{k^{2} r_{D}^{2}}\left(1+\frac{T k^{2}}{m(\omega-\Omega)^{2}}\right) \\
& \times \frac{T s h(\hbar \Omega / T)}{\hbar \Omega}, \quad 1 \gg \frac{T k^{2}}{m(\omega-\Omega)^{2}},
\end{aligned}
$$


$B_{1}(k, \omega, Z)$ and $B_{2}(k, \omega, Z)$ slightly depend on the frequency. In the present conditions, it occurs that $B_{2}(k, \omega, Z) \gg$ $B_{1}(k, \omega, Z)$. That is why $B_{1}(k, \omega, Z)$ can be taken into account by the perturbation technique. The only one solution of (58) satisfies inequalities (59). As a result, we have

$$
\omega_{1}^{2}=\Omega^{2} \frac{2 Z}{k^{2} r_{D}^{2}} \frac{T s h(\hbar \Omega / T)}{\hbar \Omega}, \quad k^{2} r_{D}^{2} \ll Z \ll 1
$$

So, taking into account inequality $Z \neq 0$ at small $k$, we state that the Vlasov spectrum (57) changes significantly.

The more important fact is the depending of the result through $Z$ on Planck constant $\hbar$. The larger is $Z$, the larger is the region of validity of the formula (60).

Let now $Z \ll k^{2} r_{D}^{2} \ll 1$. The equation describing the oscillation spectrum has the form:

$$
\begin{aligned}
1 & -\frac{B_{1}(k, \omega, Z)}{\omega^{2}}-\frac{e^{2}}{k^{2} \hbar V} \sum_{\mathbf{p}} e^{-\left(E^{(0)}-\mu_{0}\right) / T} \frac{2 Z \Omega}{(\omega-(\mathbf{p k} / m))^{2}-\Omega^{2}} \\
& \times \operatorname{sh} \frac{\hbar \Omega}{T}=0 .
\end{aligned}
$$

In this equation, the last term can be taken into account using perturbation technique. It yields

$$
\begin{gathered}
\omega_{2}^{2}=\Omega^{2}\left(1+3 k^{2} r_{D}^{2}\right)(1-2 Z) e^{\left(\mu-\mu_{0}\right) / T} \\
\times\left(1+\frac{2 Z}{k^{2} r_{D}^{2}} \frac{\Omega^{2}}{\sqrt{2 \pi m T}} \frac{T s h(\hbar \Omega / T)}{\hbar \Omega} P\right. \\
\times \int_{-\infty}^{\infty} \frac{\exp \left(-p_{z}^{2} / 2 m T\right) d p_{z}}{3 \Omega^{2} k^{2} r_{D}^{2}-2 \Omega\left(p_{z} k / m\right)} \\
\left.-i \pi \frac{Z}{k^{2} r_{D}^{2} \sqrt{2 \pi}} \frac{T s h(\hbar \Omega / T)}{\hbar \Omega}\right), \\
Z \ll k^{2} r_{D}^{2} \ll 1 .
\end{gathered}
$$

With respect to (60), it is another brunch of spectrum. The symbol $P$ means the principal value of the integral. The expression (62) is written in the form, which allows comparison with Vlasov formula (57). It is evident that in the region under consideration the Vlasov formula is correct but the presence of parameter $Z \neq 0$ means that all the oscillation modes through imagine part Im $\omega_{2}^{2}$ are damped. Moreover, the dispersion condition (57) can hardly be implemented in practice. In real electron-ion low-temperature plasma condition is always $Z \gg 1$.

Now, we consider the more important case when $Z \gg 1$. The equation describing the oscillation spectrum reads

$$
1-\frac{B_{1}(k, \omega, Z)}{\omega^{2}}-\frac{B_{3}(k, \omega, Z)}{\omega^{2}-4 \Omega^{2}(1+Z)}=0,
$$

where

$$
\begin{gathered}
B_{1}(k, \omega, Z)=\frac{\Omega^{2}}{2}\left(1+3 \frac{T k^{2}}{m \omega^{2}}\right) e^{\left(\mu-\mu_{0}\right) / T}, \quad 1 \gg 3 \frac{T k^{2}}{m \omega^{2}}, \\
B_{3}(k, \omega, Z)=\frac{2 \Omega^{2} Z}{k^{2} r_{D}^{2}} e^{\left(\mu-\mu_{0}\right) / T} \frac{T t h(\hbar \Omega \sqrt{Z} / T)}{\hbar \Omega \sqrt{Z}} \\
\times\left(1+\frac{T k^{2}}{m(\omega-2 \Omega \sqrt{Z})^{2}}\right), \\
1 \gg \frac{T k^{2}}{m(\omega-2 \Omega \sqrt{Z})^{2}} .
\end{gathered}
$$

Also, (63) describes two spectrum branches:

$\omega_{3,4}^{2}=\frac{1}{2}\left(4 \Omega^{2} Z+B_{1}+B_{3}\right) \pm \sqrt{\frac{1}{4}\left(4 \Omega^{2} Z-B_{1}+B_{3}\right)^{2}+B_{1} B_{2}}$,

as a solution of biquadrate equation. In conditions under consideration, the following inequality is correct $B_{3}(k, \omega, Z) \gg B_{1}(k, \omega, Z)$. The only one result coinciding with inequalities (64) follows:

$$
\begin{array}{r}
\omega_{3}^{2}=4 \Omega^{2} Z\left(\frac{1}{2 k^{2} r_{D}^{2}} e^{\left(\mu-\mu_{0}\right) / T} \frac{T \operatorname{th}(\hbar \Omega \sqrt{Z} / T)}{\hbar \Omega \sqrt{Z}}+1\right), \\
k^{2} r_{D}^{2} \ll \sqrt{Z} .
\end{array}
$$

This formula differs significantly from Vlasov's formula (57). We state that for the real conditions $Z \gg 1$ the presence (63) of the second term dramatically changes the Vlasov result. This branch of spectrum depends principally on $\hbar$ since $Z \sim 1 / \hbar^{2}$.

\section{Debye Screen Radius}

Equation (50) can be cast in the form:

$$
k^{2} \varepsilon^{l}(k, \omega) \varphi_{\mathrm{cl}}(\mathbf{k}, \omega)=\rho_{\mathrm{cl}}(\mathbf{k}, \omega),
$$

where $\rho_{\mathrm{cl}}(\mathbf{k}, \omega)$ is the density of external classical charge present in plasma and

$$
\varphi_{\mathrm{cl}}(\mathbf{r}, t)=\int e^{i k r-i \omega t} \frac{\rho_{\mathrm{cl}}(\mathbf{k}, \omega)}{k^{2} \varepsilon^{l}(\mathbf{k}, \omega)} \frac{d \mathbf{k} d \omega}{(2 \pi)^{4}} .
$$

If the point charge $Q$ is placed at point $\mathbf{r}=0$ and does not depend on time, then $\rho_{\mathrm{cl}}(\mathbf{k}, \omega)=2 \pi \delta(\omega) Q$. Using $(68)$ at $Z=0$, one gets the conventional result:

$$
\varphi_{\mathrm{cl}}(\mathbf{r})=\frac{Q}{4 \pi r} e^{-r / r_{D}}
$$


According to (53) at arbitrary $Z$ and $k \rightarrow 0$,

$$
\begin{aligned}
\mathcal{E}^{l}(\mathbf{k}, 0)=1 & +\frac{1}{k^{2} r_{D}^{2}} e^{\left(\mu-\mu_{0}\right) / T} \\
\times & {\left[\frac{1}{(1+Z)^{2}}+\frac{1}{2}\left(\frac{Z}{1+Z}\right)^{2} \operatorname{ch} \frac{\hbar \Omega \sqrt{1+Z}}{T}\right.} \\
& +Z \frac{2 T \sqrt{1+Z}}{\hbar \Omega(1+Z)^{3}} \operatorname{sh} \frac{\hbar \Omega \sqrt{1+Z}}{T} \\
+ & \left.\frac{2 Z}{(1+Z)^{3}} e^{\left(\mu-\mu_{0}\right) / T} \frac{T \sqrt{1+Z}}{2 \hbar \Omega(1+Z)^{3}} \operatorname{sh} \frac{\hbar \Omega \sqrt{1+Z}}{T}\right] \\
\times & {\left[1+\frac{T}{\hbar \Omega \sqrt{1+Z}}\left(1+\frac{Z}{16}\right) \operatorname{sh} \frac{\hbar \Omega \sqrt{1+Z}}{T}\right] . }
\end{aligned}
$$

The term multiplied by $1 / k^{2} r_{D}^{2}$ describes the Debye radius renormalization. The last term takes part in radius $r_{D}$ and charge $Q$ renormalization. If $Z \gg 1$, then using (36):

$$
\varepsilon^{l}(\mathbf{k}, 0)=1+\frac{1}{2 k^{2} r_{D}^{2}}\left(1+\frac{T}{\hbar \Omega \sqrt{Z}} t h \frac{\hbar \Omega \sqrt{Z}}{T}\right),
$$

and as a consequence, we return for $r \rightarrow \infty$ to the formula (69) in which now instead of $r_{D}$ the value $r_{D}^{*}$ is placed where

$$
\begin{aligned}
r_{D}^{*} & =2 r_{D}\left(1+\frac{T}{\hbar \Omega \sqrt{Z}} t h \frac{\hbar \Omega \sqrt{Z}}{T}\right)^{-1} \\
& \approx r_{D}\left(1-\frac{1}{12 \pi^{2}} \frac{e^{2}}{r_{D} T}\right) \text { since } \frac{e^{2}}{r_{D} T} \ll 1 .
\end{aligned}
$$

This formula does not depend on $\hbar$. Such a fact once again manifests the "camouflage effect" explaining in some cases the pseudoreasonable results following from conventional classical theory of plasma.

\section{Conclusion}

The electromagnetic field is always quantized. The question is whether the quantum properties of the field are significant or not in a particular system.

In the present work, we show that as quantization procedure of longitudinal (Langmuir) electromagnetic field and taking into account the electron interaction with RayleighJeans distribution of Langmuir waves at finite temperature change dramatically the structure of the electron cloud in a low-temperature plasma. The dimensionless quantum parameter $Z$ is proportional to the charge squared whose value is much larger than the one appears in theory. It makes impossible to use a perturbation technique. We tried to construct the closed system of equations, the solution of which using perturbation technique (small $Z$ ) returns to the consequences of Vlasov equation. In real conditions of the low-temperature electron-ion plasma, $Z \gg 1$ that is why, in any case, the plasma optical properties could not be investigated using Vlasov's equation. The analysis of the obtained system of equations at large $Z$ leads to the conclusion that the quantum properties of plasmas manifest themselves dramatically in kinetic and hydrodynamic properties.

\section{References}

[1] L. Tonks and I. Langmuir, "Oscillations in ionized gases," Physical Review, vol. 33, no. 2, pp. 195-210, 1929.

[2] A. A. Vlasov, "On vibration properties of electron gas," Journal of Experimental and Theoretical Physics, vol. 8, no. 3, p. 291, 1938 (Russian).

[3] L. D. Landau, "O kolebaniiakh elektronnoi plazmy," Journal of Experimental and Theoretical Physics, vol. 16, p. 574, 1946 (Russian).

[4] A. F. Alexandrov, L. S. Bogdankevich, and A. A. Rukhadze, Principles of Plasma Electrodynamics, Springer, Berlin, Germany, 1984.

[5] P. K. Shukla and B. Eliasson, "Nonlinear aspects of quantum plasma physics," Uspekhi Fizicheskikh Nauk, vol. 180, pp. 55-82, 2010.

[6] R. Glauber, in Quantum Optics and Electronics, C. DeWitt, A. Blandin, and C. Cohen-Tannoudji, Eds., Universite deGrenoble. Ecole d'ete Physique Theorique 1964.Gordon and Breach, Science Publishers, New York, NY, USA, 1965.

[7] M. V. Kuselev and A. A. Rukhadze, "On the quantum description of the linear kinetics of a collisionless plasma," PhysicsUspekhi, vol. 42, no. 6, p. 603, 1999.

[8] V. L. Bonch-Bruevich and S. V. Tjablikov, The Method of Green Functions in Statistical Mechanics, URSS, Moscow, Russian, 1961.

[9] H. B. Callen and T. A. Welton, "Irreversibility and generalized noise," Physical Review, vol. 83, no. 1, pp. 34-40, 1951. 

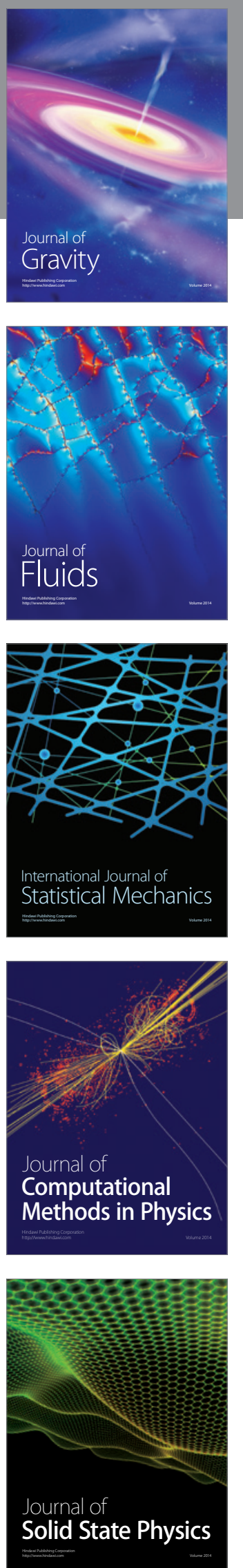

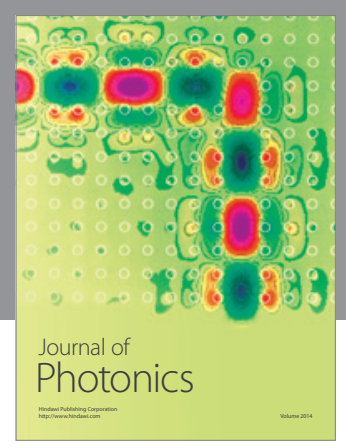

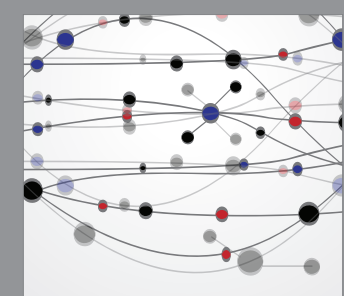

The Scientific World Journal
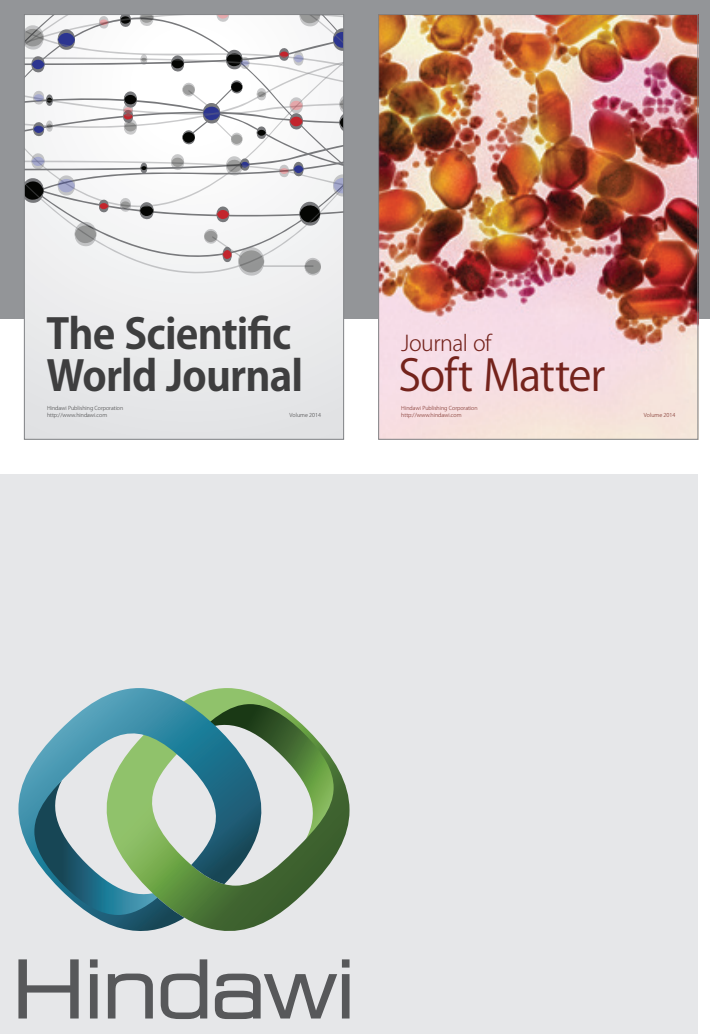

Submit your manuscripts at

http://www.hindawi.com
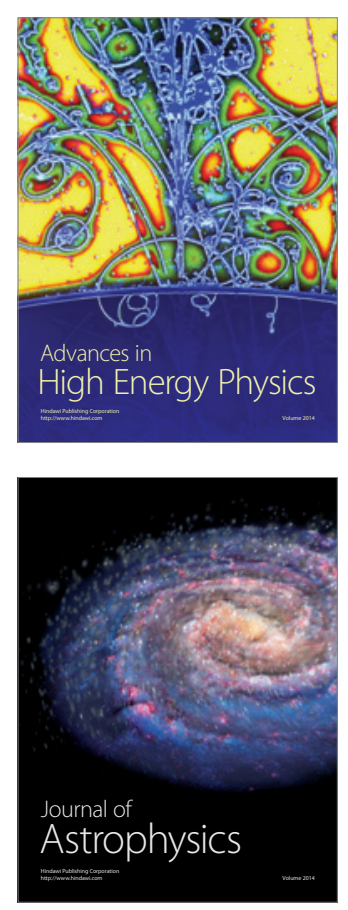
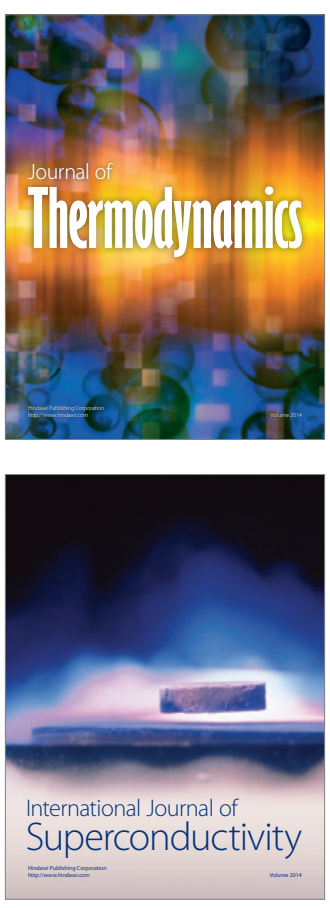
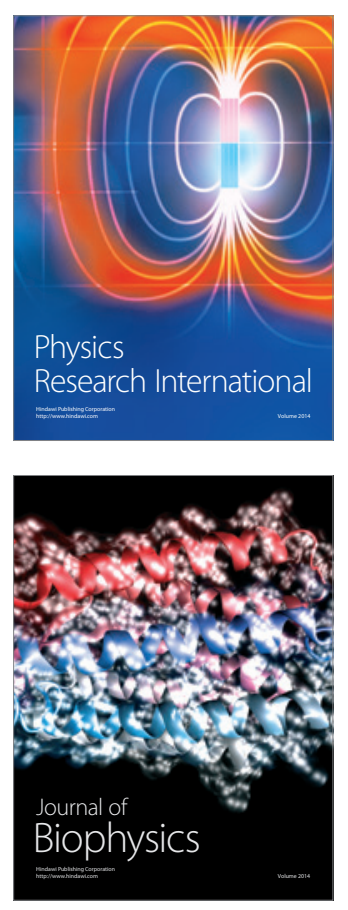
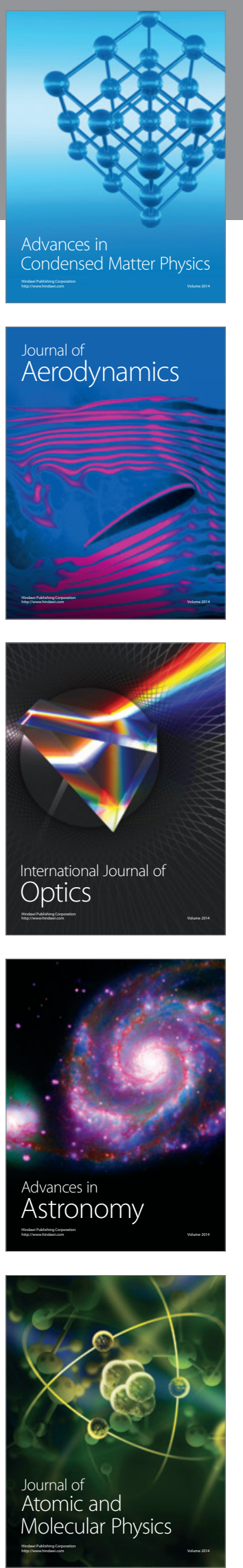